ARTICLE

Check for updates

https://doi.org/10.1038/s41467-021-22808-7

OPEN

\title{
Interconversion of multiferroic domains and domain walls
}

\author{
E. Hassanpour (10 1,2,7, M. C. Weber (1) 1,7凶, Y. Zemp ${ }^{1}$, L. Kuerten (1) ${ }^{1}$, A. Bortis (1) ${ }^{1}$, Y. Tokunaga ${ }^{3}$, Y. Taguchi (1) ${ }^{4}$, \\ Y. Tokura (i) ${ }^{4,5}$, A. Cano (1) ${ }^{1,6}$, Th. Lottermoser (1) ${ }^{1} \&$ M. Fiebig (i) ${ }^{1}$
}

Systems with long-range order like ferromagnetism or ferroelectricity exhibit uniform, yet differently oriented three-dimensional regions called domains that are separated by twodimensional topological defects termed domain walls. A change of the ordered state across a domain wall can lead to local non-bulk physical properties such as enhanced conductance or the promotion of unusual phases. Although highly desirable, controlled transfer of these properties between the bulk and the spatially confined walls is usually not possible. Here, we demonstrate this crossover by confining multiferroic $\mathrm{Dy}_{0.7} \mathrm{~Tb}_{0.3} \mathrm{FeO}_{3}$ domains into multiferroic domain walls at an identified location within a non-multiferroic environment. This process is fully reversible; an applied magnetic or electric field controls the transformation. Aside from expanding the concept of multiferroic order, such interconversion can be key to addressing antiferromagnetic domain structures and topological singularities.

\footnotetext{
${ }^{1}$ Department of Materials, ETH Zurich, Zurich, Switzerland. ${ }^{2}$ Department of Physics, ETH Zurich, Zurich, Switzerland. ${ }^{3}$ Department of Advanced Materials Science, University of Tokyo, Kashiwa, Japan. ${ }^{4}$ RIKEN Center for Emergent Matter Science (CEMS), Wako, Japan. ${ }^{5}$ Department of Applied Physics, University of Tokyo, Tokyo, Japan. ${ }^{6}$ Univ. Grenoble Alpes, CNRS, Grenoble INP, Institut Néel, Grenoble, France. ${ }^{7}$ These authors contributed equally: E. Hassanpour, M. C. Weber. ${ }^{凶}$ email: mads.weber@mat.ethz.ch
} 
R ecently, the interests in ferroic materials with magnetic or electric order evolved from the spatially expanded bulk domains towards the spatially confined domain walls. As inherent inhomogeneity, domain walls are a source of specific phenomena that are forbidden in the uniform interior of the corresponding domains ${ }^{1,2}$. Examples are the occurrence of magnetization, polarization, magnetoelectric coupling, (super-) conductivity, memory effects, or a change of melting temperature ${ }^{3-15}$. Hence, domain walls may be regarded as additional state of a material, virtually a world in its own self and often discussed without reference to the surrounding bulk phase.

It would be very desirable if the dimensional limitation of these phenomena could be overcome. For example, one could consider the confinement of a multifunctional bulk state into domain walls where they could establish a form of rewritable electromagnetic circuits $^{5,16}$. Reversely, the domain walls may seed the recovery of the original bulk state, acting as its memory ${ }^{10,17}$.

A class of materials in which the transition from bulk domains to domain walls could be virtually continuous are compounds with phase transitions, in which the symmetry of the domains and domain walls coincide crosswise on either sides of the phase boundary. In such materials, domain walls can in principle gradually transform into domains, and vice versa, across a first-order phase transition. This concept was discussed in theoretical physics ${ }^{18-20}$, and systems showing a behavior consistent with this concept were reported ${ }^{21,22}$. Conclusive observation of a smooth, deterministic transfer of a domain wall into a domain, however, and, in particular, the reversible interconversion between domains and domain walls have not been presented. Notably, the practical consequences of such interconversion for the physical properties and functionalities of materials were never debated.

We demonstrate this interconvertibility and apply it to tune a multiferroic state between three and two dimensions in a controlled way. Specifically, we confine a multiferroic bulk state into a multiferroic domain wall at an identified location within a nonmultiferroic environment. We act on this spatially confined magnetoelectric object with magnetic or electric fields and evidence the presence of a switchable magnetization and polarization in the wall. We furthermore employ the fields to transfer the multiferroic domain wall back into a multiferroic bulk state. We then discuss the general occurrence and benefits of an ordered state with controllable transfer in between spatial expansion and spatial confinement.

\section{Results and discussion}

For the reasons given below, we choose the rare-earth orthoferrite $\mathrm{Dy}_{0.7} \mathrm{~Tb}_{0.3} \mathrm{FeO}_{3}$ (see elsewhere ${ }^{23}$ about sample preparation) as our model system. Multiferroicity occurs at $2.65 \mathrm{~K}$, caused by simultaneous antiferromagnetic ordering of the rare-earth and iron spin systems, coupled by exchange striction ${ }^{23}$. An electric polarization $P_{\mathrm{s}}=0.12 \mu \mathrm{C} \mathrm{cm}^{-2}$ coexists with a Dzyaloshinskii-Moriya-type weak magnetization $M_{\mathrm{s}}=0.15 \mu_{\mathrm{B}}$ per formula unit, both oriented along the $c$-axis. Subsequently, at $T_{\mathrm{C}} \simeq 2.3 \mathrm{~K}$ a first-order spin reorientation of the iron sublattice from the multiferroic to an antiferromagnetic phase with $M_{\mathrm{s}}, P_{\mathrm{s}}=0$ occurs. The simplest possible form to describe such a transition in between two

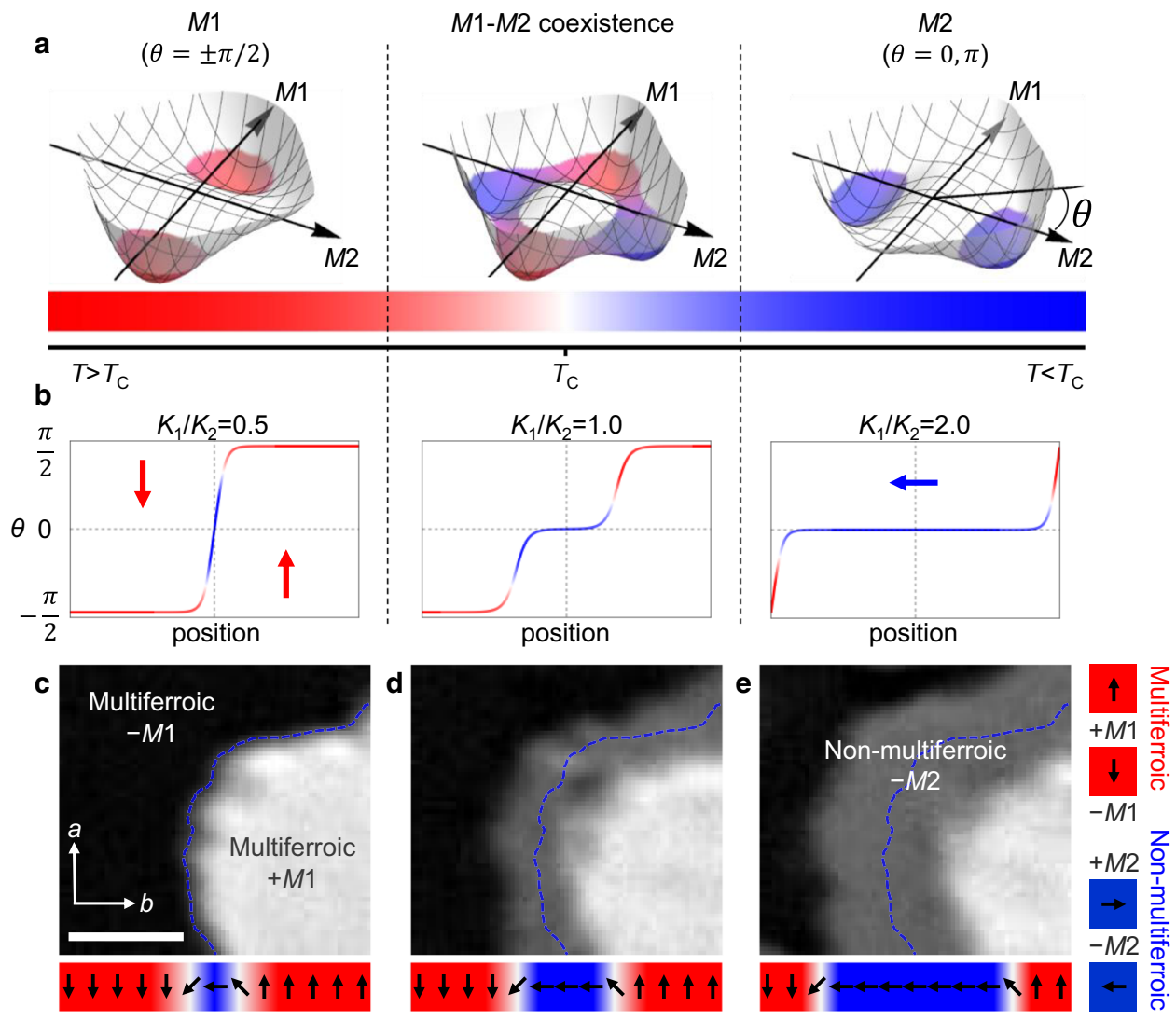

Fig. 1 Interconversion of domains and domain walls. a Temperature dependence of the free-energy landscape for the first-order phase transition of Eq. (1) with $\theta$ as orientation of the order parameter. $\mathbf{b}$ Evolution of domains and domain walls across the $M 1 \leftrightarrow M 2$ phase transition. Domain walls expand into domains and domains are confined into domain walls, with an intermediate regime where the $M 1$ and $M 2$ phases coexist. $K_{1}$ and $K_{2}$ are the magnetocrystalline anisotropy parameters of Eq. (1). Blue and red arrows depict the orientation $\theta$ of the order parameter. c-e Upon temperature decrease, a domain wall (dashed blue line) separating opposite multiferroic domains in c expands and transforms to a domain of the non-multiferroic phase in $\mathbf{c}$ and $\mathbf{d}$. Faraday rotation (FR) by $\phi_{\mathrm{FR}}$ shows the $M 1$ domains as dark $\left(\phi_{\mathrm{FR}}<0\right)$ and bright $\left(\phi_{\mathrm{FR}}>0\right)$ regions and the M2 domains $\left(\phi_{\mathrm{FR}}=0\right)$ as gray area. Scale bar, $100 \mu \mathrm{m}$. 
a

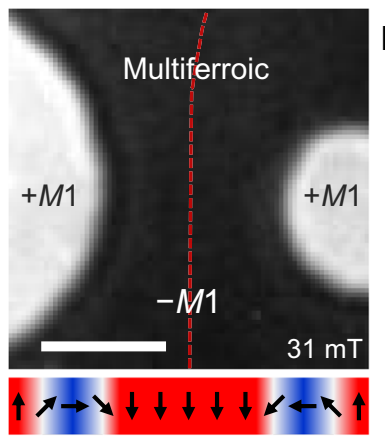

d

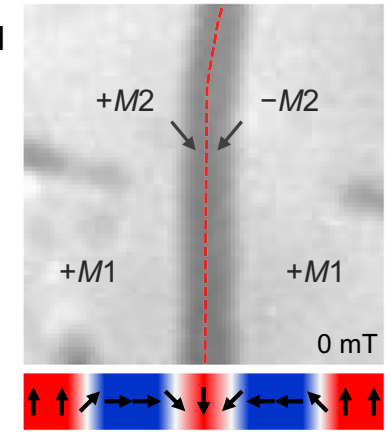

b

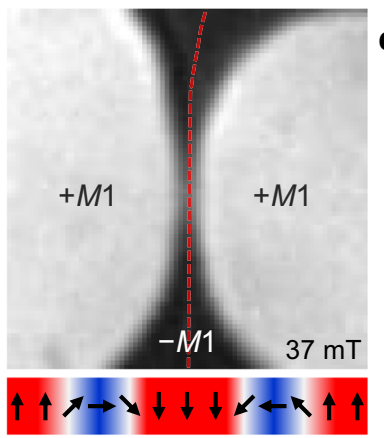

Multiferroic
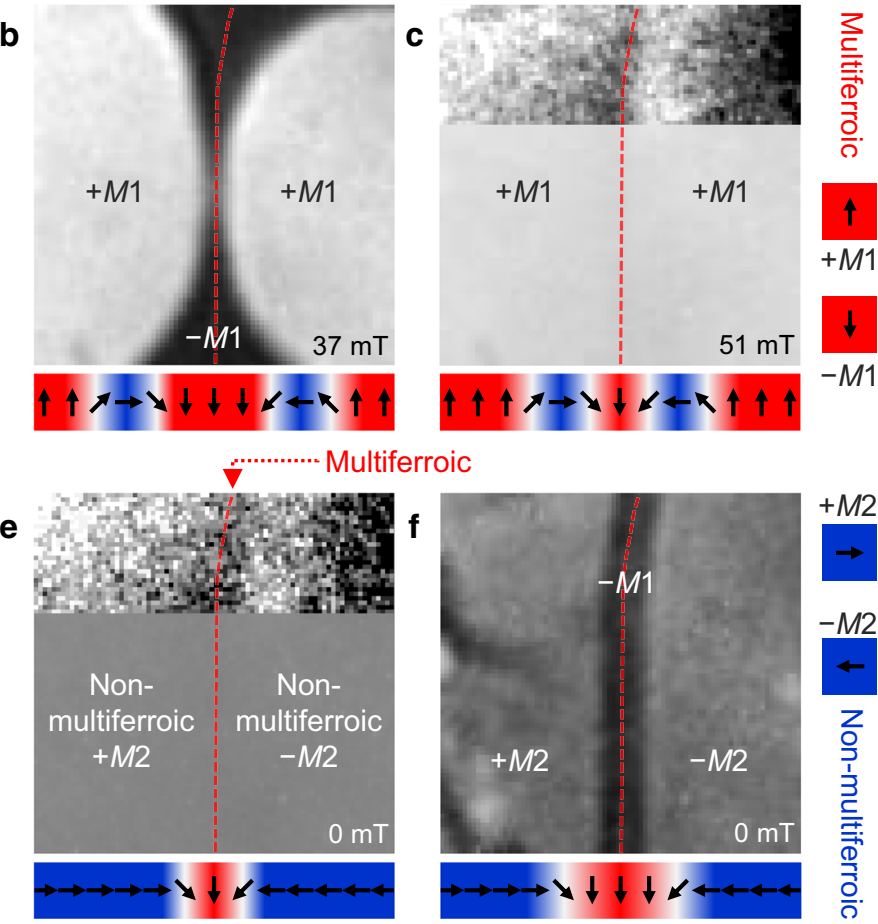

Fig. 2 Transformation of a multiferroic bulk domain into a multiferroic domain wall in a non-multiferroic environment. Panels a-f show the same area, using the same gray scale (except in the insets of $\mathbf{c}$ and $\mathbf{e}$, which are rescaled to minimum = black, maximum $=$ white). Arrows indicate the orderparameter orientation (see Fig. 1a) for the horizontal succession of phases in the associated images. a-c Starting from a single-domain state ( - M1, dark), two opposite multiferroic domains ( $+M 1$, bright) are generated by application of an out-of-plane magnetic field $H$. The $+M 1$ domains grow until they meet along the red dashed line. Here, the two domain walls in $\mathbf{a}$ and $\mathbf{b}$ have merged into a single topological object, which becomes visible in the inset. d, e Setting $T=1.8 \mathrm{~K}$ at $H=0$ returns the sample to the non-multiferroic $M 2$ state. The wall separating the $+M 1$ domains in $\mathbf{c}$ expands into opposite $M 2$ domains (gray) separated by a multiferroic domain wall that becomes visible in the inset. $\mathbf{f}$ Heating the sample to $2.3 \mathrm{~K}$ lets the multiferroic domain wall in e expand into a multiferroic $-M 1$ bulk domain. Scale bar, $100 \mu \mathrm{m}$.

magnetic phases $M 1$ and $M 2$ is the phenomenological effective Hamiltonian ${ }^{19,20}$

$$
H=A|\nabla \theta|^{2}+K_{1} \sin ^{2} \theta+K_{2} \sin ^{4} \theta .
$$

As depicted in Fig. 1, the angle $\theta$ distinguishes the domain states on the two sides of the $M 1 \leftrightarrow M 2$ phase transition. $K_{1}$ and $K_{2}$ are magnetocrystalline anisotropy parameters. $K_{1}=0$ and $K_{1}=-2 K_{2}$ set the limits of the phase-coexistence region of $M 1$ and $M 2$. The gradient term with $A$ as exchange constant further determines the order-parameter rotation across the domain walls.

In $\mathrm{Dy}_{0.7} \mathrm{~Tb}_{0.3} \mathrm{FeO}_{3}, M 1$ and $M 2$ are associated with the multiferroic (weakly ferromagnetic) and non-multiferroic (purely antiferromagnetic) phases, respectively, with $\theta_{ \pm M 1}=+\pi / 2,-\pi / 2$ and $\theta_{ \pm M 2}=0, \pi$. Figure $1 \mathrm{~b}$ depicts the evolution according to Eq. (1) of a configuration starting with two well-defined domains outside the coexistence region and separated by a single domain wall ${ }^{18}$. By entering the $M 1 / M 2$ coexistence region, the domain wall widens and transforms into a domain of the other phase. Conversely, the initial domain shrinks and eventually transforms into a domain wall separating the domains on the opposite side of the coexistence region. Hence, the first-order phase transition between the multiferroic M1 and the non-multiferroic $M 2$ phase in $\mathrm{Dy}_{0.7} \mathrm{~Tb}_{0.3} \mathrm{FeO}_{3}$ should in principle allow us to reversibly convert a multiferroic domain into a multiferroic domain wall in a non-multiferroic environment.

We first verify the theoretically predicted transformation of a domain wall into a domain ${ }^{18}$ experimentally. In $\mathrm{Dy}_{0.7} \mathrm{~Tb}_{0.3} \mathrm{FeO}_{3}$, we can exploit the magnetization of the multiferroic phase to distinguish the $M 1$ and $M 2$ domain states by spatially resolved real-time imaging experiments, using the magneto-optical Faraday effect as magnetization probe. Furthermore, $\mathrm{Dy}_{0.7} \mathrm{~Tb}_{0.3} \mathrm{FeO}_{3}$ exhibits a relatively broad region of phase coexistence. This gives us ample time to image the phase coexistence while tuning the balance between the competing multiferroic and non-multiferroic phases.

Figure $1 \mathrm{c}-\mathrm{e}$ shows sequential Faraday images and the associated order-parameter distribution of a $c$-oriented $\mathrm{Dy}_{0.7} \mathrm{~Tb}_{0.3} \mathrm{FeO}_{3}$ sample cooled across the $M 1 \leftrightarrow M 2$ transition. We refrain from quantifying $K_{1}$ and $K_{2}$ since their absolute values are not relevant for our discussion. In Fig. $1 \mathrm{c}$ we see a $\pm M 1$ domain pair at $T>T_{\mathrm{C}}$. Because of the opposite direction of magnetization and, hence, Faraday rotation, the domains appear as bright and dark regions. Figure $1 \mathrm{~d}$, e show the same region about 4 and 8 min after cooling the sample to $T \lesssim T_{\mathrm{C}}$. A gray stripe centers at the domain-wall position of Fig. 1c that widens with time. Its zero Faraday rotation identifies it as antiferromagnetic region. Below $T_{C}$, this is the dominating phase and, starting from the original $+M 1 /-M 1$ domain wall, we perceive the homogeneous expansion of this wall into an $M 2$ domain engulfing the sample as time progresses. Note that this expansion of the non-multiferroic phase occurs in a deterministic way, starting uniformly from the center of the domain wall of the multiferroic phase. This deterministic behavior is in contrast to the needle-like growth of domains of the emergent phase at domain walls of the original phase ${ }^{21}$ or to the nucleation on random defects as in most first-order phase transitions ${ }^{24}$. Defects do not play a role in our case as we experimentally verify in Supplementary Note 3. Furthermore, the deterministic expansion requires an order-to-order transition, whereas order-todisorder transitions into phase coexistence ${ }^{25}$ are not covered by Eq. (1) and must have a different origin ${ }^{18}$.

Now we employ this deterministic aspect for confining a multiferroic domain into a multiferroic domain wall at an identified location within a non-multiferroic environment. We begin at $T>T_{\mathrm{C}}$ with a $-M 1$ domain sandwiched between $+M 1$ domains (Fig. $2 a$ ). In 


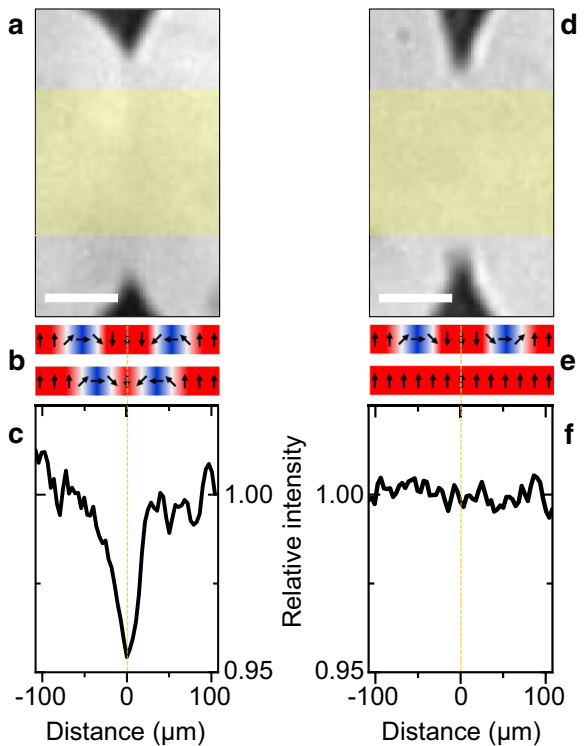

Fig. 3 Non-coalescing and coalescing multiferroic domains. a Meeting of two multiferroic $+M 1$ domains obtained following the procedure as in Fig. 2a-c. The sense of rotation of the order parameter across the $+M 1 \rightarrow$ $-M 1$ domain wall is the same for both $+M 1$ domains. $\mathbf{b}$ Order-parameter orientation (arrows) across the boundary of the $+M 1$ walls before (top) and after (bottom) the meeting of the $+M 1$ domains. $\mathbf{c}$ Horizontal intensity scan of the image in a, vertically averaged across the area hatched yellow. The intensity dip indicates the formation of a $360^{\circ}$ domain wall between the $+M 1$ domains. $\mathbf{d}-\mathbf{f}$ Same as $\mathbf{a}-\mathbf{c}$, but with opposite sense of rotation of the order parameter across the $+M 1 \rightarrow-M 1$ domain wall for the two $+M 1$ domains. Here the $+M 1$ domains coalesce into a single domain. Scale bars, $100 \mu \mathrm{m}$.

a magnetic field we enlarge the $+M 1$ domains (Fig. $2 \mathrm{~b}$ ) until they meet (Fig. 2c). After cooling the sample to $T \lesssim T_{\mathrm{C}}$, the antiferromagnetic state emerges from the spatially confined object at the meeting point of the $+M 1$ domains (Fig. $2 \mathrm{~d}$ ) and grows with time until it has filled the entire field of view (Fig. 2e).

As we see in Fig. 3, there are two types of meetings between $+M 1$ domains on a $-M 1$ background. As graphically illustrated in the Supplementary Note 4, they reflect the two types of domain walls a multiferroic domain is expected to exhibit, namely $+M 1 \rightarrow-M 1$ walls with either clockwise or counterclockwise rotation of the order parameter across the wall ${ }^{26}$. When domain walls of the opposite type meet, the respective rotations cancel and the walls annihilate so that the meeting domains coalesce. Alternatively, for a meeting of the same type of domain walls, a $360^{\circ}$ spin rotation occurs as sketched in Fig. $3 \mathrm{~b}$ and Supplementary Note 4 . This topologically protected object prevents the coalescence of the domains and is visible as brightness dip in Fig. 3c. Instead, it can seed a $+M 2$ and a $-M 2$ domain as sketched beneath Fig. $2 d$, e. Note that a magnetic field three times the value of the coercive field is required to destroy the $360^{\circ}$ wall.

Hence, Fig. 2e shows a $+M 2$ and a $-M 2$ domain; according to Fig. 1a, they are separated by a multiferroic domain wall into which the multiferroic $-M 1$ bulk state of Fig. 2a has been confined. This wall has been placed at the meeting point of the two former $+M 1$ domains in Fig. $2 c$. For confirmation, we re-heat the sample to $T \gtrsim$ $T_{C}$; Fig. $2 f$ shows that this reconverts the suspected multiferroic domain wall into a multiferroic $-M 1$ domain. Note that even though the optical resolution is not sufficient to reveal the microscopic structure of the domain wall, we still can estimate its width to be about $1 \mu \mathrm{m}$ through deconvolution of the optical signal as described in Supplementary Note 1. In complementary experiments on the closely related compound $\mathrm{DyFeO}_{3}$, we verify the value directly by magnetic force microscopy measurements in Supplementary Note 2.
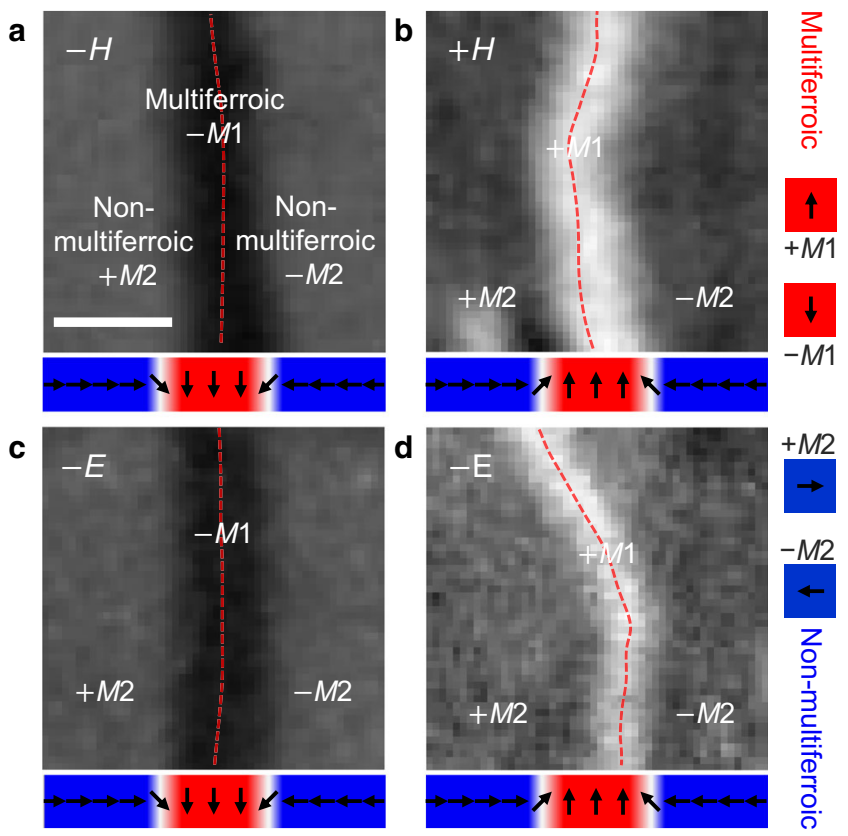

Fig. 4 Magnetoelectric field control of multiferroic domain walls in a nonmultiferroic environment. Using the same gray scale, a-d show equally sized regions on the same sample, yet imaged on different areas and in separate experiments. For experiments in $\mathbf{a}-\mathbf{c}$, a state as in Fig. 2e with $+M 2$ and $-M 2$ domains separated by a $-M 1$-like domain wall was prepared while for $\mathbf{d}$, opposite $M 2$ domains are separated by a $+M 1$-like domain wall. Magnetic and electric fields are applied as labeled, with the + sign indicating out-of-plane orientation. Beneath each image, the associated orderparameter orientation is sketched in analogy to Fig. 2. a, b A magnetic field $\pm H$ sets the magnetization of the multiferroic wall to $\pm M_{s}$ and expands it into a $\pm M 1$ multiferroic bulk domain with a 1:1 correspondence between the signs of $H$ and $M_{\mathbf{s}} . \mathbf{c}, \mathbf{d}$ An electric field $\pm E$ sets the polarization of the multiferroic wall to $\pm P_{\mathrm{s}}$ and expands it into a multiferroic $M 1$ bulk domain. Because of the involvement of three order parameters, the sign of $M_{\mathrm{s}}$ is not determined by the sign of $P_{\mathrm{s}}(E)$ but rather by the history of the sample 27 . This is highlighted by $\mathbf{c}$ and $\mathbf{d}$, where a field $-E$ induces $a+M 1$ or $-M 1$ domain, respectively. Scale bar, $30 \mu \mathrm{m}$. $\mu_{0} \mathrm{H}=0.4 \mathrm{~T} ; E=83.3 \mathrm{kV} / \mathrm{cm}$.

These measurements allow us to show the clear difference between the domain wall that we probe and the alternative case of a narrow domain.

To describe the wall as multiferroic, we furthermore have to show that we can act on it and affect its magnetization and electric polarization by an applied magnetic or electric field. This is also important for its technological utility. This is done in Fig. 4 which shows the response of a multiferroic domain wall in a nonmultiferroic environment, generated as in Fig. 2e, to static $c$ oriented magnetic or electric fields. We see that both fields initiate the transfer of the wall back into a domain of the multiferroic phase, even though temperature-wise the material still favors the non-multiferroic phase. This is only possible because the wall has a magnetization $M_{\mathrm{s}}$ and a polarization $P_{\mathrm{s}}$ the respective applied field can act on to initiate the transfer. Furthermore, the transfer is energetically beneficial only if $M_{\mathrm{s}}$ or $P_{\mathrm{s}}$ points in the direction of the applied field. Since the field has triggered the transfer in all our experiments, the wall magnetization and polarization itself must be switchable, and hence set the direction of $M_{\mathrm{s}}$ or $P_{\mathrm{s}}$ of the expanding domain. Determination of the sign of $M_{\mathrm{s}}$ by the direction of the magnetic field is evident from Fig. $4 a$, b, whereas Fig. $4 \mathrm{c}$ shows that the electric field acts on the magnetization via magnetoelectric coupling. (Note that because of the involvement of three order parameters in this coupling ${ }^{27}$, the sign of $M_{\mathrm{s}}$ is not 
determined by the sign of $P_{\mathrm{s}}(E)$ but rather by the history of the sample, as comparison of Fig. $4 c, d$ shows.) We thus see that despite the spatial confinement and the non-multiferroic environment, a wall as in Fig. 2e retains the magnetoelectric manipulability of the multiferroic bulk phase.

To conclude, we have experimentally demonstrated the conversion of a multiferroic bulk phase into a multiferroic domain wall at an identified location inside a non-multiferroic environment. The initial multiferroic hallmark properties, namely a magnetization and a polarization that are coupled and switched by an applied field, are retained by the wall. Magnetic or electric fields act on the wall and convert it back into a bulk multiferroic domain with a predetermined direction of magnetization or polarization.

The concept of interconversion can be expanded beyond multiferroics. First, there are plenty of first-order magnetic phase transitions fulfilling the rather basic conditions of Eq. (1) with $\mathrm{CaFe}_{2} \mathrm{O}_{4}$ as a very recent example to catch scientific attention ${ }^{28}$. Note that even if the region of phase coexistence may be narrower than in our case, possibly even too narrow for convenient experimental probing during the transition, a system will still be subject to the same dynamics as found in $\mathrm{Dy}_{0.7} \mathrm{~Tb}_{0.3} \mathrm{FeO}_{3}$. Even non-magnetic types of order can display the spatial interconversion. For example, a (static) expansion of domain walls into domains with phase coexistence based on a chemical gradient rather than the first-order nature of a phase transition was observed in the ferroelectric-to-nonferroelectric transition in $\mathrm{InMnO}_{3}{ }^{29}$. Even organic materials may show a transition between bulk phases with neutral and ionic building blocks emerging out of an expanding singularity by progressive charge transfer ${ }^{30,31}$.

Second, our work expands the possibilities for functionalizing domain walls. Unlike in previous examples ${ }^{14,15}$, the magnetoelectric properties are not confined to the domain walls but can be expanded into the bulk when needed. The magnetoelectric properties of the walls may be employed to visualize or even tailor domain patterns in materials whose order is normally difficult to access, such as $180^{\circ}$ antiferromagnets. Finally, our work provides a rare opportunity of deterministic nucleation in a first-order phase transition since the nucleating phase is seeded by the order and symmetry in domain walls rather than occurring on random defects.

\section{Methods}

Single-crystals of $\mathrm{Dy}_{0.7} \mathrm{~Tb}_{0.3} \mathrm{FeO}_{3}$ were grown by the floating-zone method under $\mathrm{O}_{2}$ atmosphere. Using back-reflection X-ray Laue diffractometry, thin $c$-oriented plates of $2.5 \times 2.5 \mathrm{~mm}^{2}$ were cut, lapped, and polished with a silica slurry down to $\sim 60-80 \mu \mathrm{m}$ with optically flat surfaces on both sides. Temperature and magnetic field treatment was done in a liquid-helium-cooled magnetic-field cryostat (Oxford Spectromag 4000-10T). For Faraday imaging, a light-emitting diode with a central wavelength of $660 \mathrm{~nm}$ (Thorlabs M660L4) was used along with a monochromatic digital camera (The Imaging Source DMK 22BUC03) for image acquisition. Image processing is limited to adjustments of brightness, contrast and levels and homogeneously applied to the entire image, unless noted. The images of each figure were adjusted equally. For electric field measurements, 3-nm platinum electrodes were deposited on both surfaces of the specimen.

Reporting summary. Further information on research design is available in the Nature Research Reporting Summary linked to this article.

\section{Data availability}

The data that support the findings of this study are available from the corresponding author on request.

Received: 19 February 2021; Accepted: 29 March 2021;

Published online: 12 May 2021

\section{References}

1. Tagantsev, A. K., Cross, L. E. \& Fousek, J. Domains in Ferroic Crystals and Thin Films. (Springer New York, 2010).
2. Hubert, A. \& Schäfer, R. Magnetic Domains. (Springer, 1998).

3. Farokhipoor, S. et al. Artificial chemical and magnetic structure at the domain walls of an epitaxial oxide. Nature 515, 379-383 (2014).

4. Van Aert, S. et al. Direct observation of ferrielectricity at ferroelastic domain boundaries in $\mathrm{CaTiO}_{3}$ by electron microscopy. Adv. Mater. 24, 523-527 (2012).

5. Aird, A., Domeneghetti, M. C., Mazzi, F., Tazzoli, V. \& Salje, E. K. H. Sheet superconductivity in $\mathrm{WO}_{3-x}$ : crystal structure of the tetragonal matrix. J. Phys. Condens. Matter 10, L569-L574 (1998).

6. Fiebig, M., Lottermoser, T., Fröhlich, D., Goltsev, A. V. \& Pisarev, R. V. Observation of coupled magnetic and electric domains. Nature 419, 818-820 (2002).

7. Seidel, J. et al. Conduction at domain walls in oxide multiferroics. Nat. Mater. 8, 229-234 (2009).

8. Meier, D. et al. Anisotropic conductance at improper ferroelectric domain walls. Nat. Mater. 11, 284-288 (2012).

9. Schröder, M. et al. Conducting domain walls in lithium niobate single crystals. Adv. Funct. Mater. 22, 3936-3944 (2012).

10. Beilsten-Edmands, J. et al. Polarization memory in the nonpolar magnetic ground state of multiferroic $\mathrm{CuFeO}_{2}$. Phys. Rev. B 94, 144411 (2016).

11. Salje, E. K. H. Multiferroic domain boundaries as active memory devices: Trajectories towards domain boundary engineering. ChemPhysChem 11, 940-950 (2010).

12. Catalan, G., Seidel, J., Ramesh, R. \& Scott, J. F. Domain wall nanoelectronics. Rev. Mod. Phys. 84, 119-156 (2012).

13. Meier, D. Functional domain walls in multiferroics. J. Phys. Condens. Matter 27, 463003 (2015).

14. Logginov, A. S., Meshkov, G. A., Nikolaev, A. V. \& Pyatakov, A. P. Magnetoelectric control of domain walls in a ferrite garnet film. JETP Lett. 86, 115-118 (2007).

15. Logginov, A. S. et al. Room temperature magnetoelectric control of micromagnetic structure in iron garnet films in iron garnet films. Appl. Phys. Lett. 93, 182510 (2017)

16. Crassous, A. et al. Nanoscale electrostatic manipulation of magnetic flux quanta in ferroelectric/superconductor $\mathrm{BiFeO}_{3} / \mathrm{YBa}_{2} \mathrm{Cu}_{3} \mathrm{O}_{7-\delta}$ heterostructures. Phys. Rev. Lett. 107, 247002 (2011).

17. Taniguchi, K., Abe, N., Ohtani, S. \& Arima, T. Magnetoelectric memory effect of the nonpolar phase with collinear spin structure in multiferroic $\mathrm{MnWO}_{4}$. Phys. Rev. Lett. 102, 147201 (2009).

18. Mitsek, A. I., Gaidanskii, P. F. \& Pushkar, V. N. Domain structure of uniaxial antiferromagnet. The problem of nucleation. Phys. Stat. Sol. 38, 69-79 (1970).

19. Belov, K. P., Zvezdin, A. K., Kadomtseva, A. M. \& Levitin, R. Z. Spinreorientation transitions in rare-earth magnets. Sov. Phys. Uspekhi 19, 574-596 (1976)

20. Bar'yakhtar, V. G., Bogdanov, A. N. \& Yablonskii, D. A. The physics of magnetic domains. Sov. Phys. Uspekhi 31, 810-835 (1988).

21. Gnatchenko, S. L., Eremenko, V. V. \& Kharchenko, N. F. Antiferromagnetic domain boundary as a line for nucleation of a weakly ferromagnetic phase from the antiferromagnetic one in dysprosium orthoferrite. Sov. J. Low Temp. Phys. 7, 742-746 (1981).

22. Kharchenko, N. F., Gnatchenko, S. L. \& Chizhik, A. B. Formation kinetics of a weak ferromagnetic domain from an AFM domain wall in $\mathrm{DyFeO}_{3}$. J. Phys. 50, 1153-1156 (1989).

23. Tokunaga, Y., Taguchi, Y., Arima, T. \& Tokura, Y. Electric-field-induced generation and reversal of ferromagnetic moment in ferrites. Nat. Phys. 8 , 838-844 (2012).

24. King, A. R. \& Paquette, D. Spin-flop domains in $\mathrm{MnF}_{2}$. Phys. Rev. Lett. 30, 662-666 (1973).

25. Dillon, J. F., Chen, E. Y., Giordano, N. \& Wolf, W. P. Time-reversed antiferromagnetic states in dysprosium aluminum garnet. Phys. Rev. Lett. 33, 98-101 (1974).

26. Houchmandzadeh, B., Lajzerowicz, J. \& Salje, E. K. H. Order parameter coupling and chirality of domain walls. J. Phys. Condens. Matter 3, 5163-5169 (1991)

27. Tokunaga, Y. et al. Composite domain walls in a multiferroic perovskite ferrite. Nat. Mater. 8, 558-562 (2009).

28. Stock, C. et al. Orphan spins in the $S=5 / 2$ antiferromagnet $\mathrm{CaFe}_{2} \mathrm{O}_{4}$. Phys. Rev. Lett. 119, 257204 (2017).

29. Huang, F.-T. et al. Duality of topological defects in hexagonal manganites Phys. Rev. Lett. 113, 267602 (2014).

30. Nagaosa, N. \& Takimoto, J. Theory of neutral-ionic transition in organic crystals. II. Effect of the intersite Coulomb interaction. J. Phys. Soc. Jpn. 55, 2745-2753 (1986)

31. Horiuchi, S., Kumai, R., Okimoto, Y. \& Tokura, Y. Neutral-ionic phase separation and one-dimensional ferroelectricity in organic relaxors. Phys. Rev. Lett. 85, 5210-5213 (2000).

\section{Acknowledgements}

We thank Dr. Morgan Trassin for fruitful discussions and for deposition of Pt electrodes on the samples. This work was financially supported by SNSF (Grant No. 200021_178825/1) and European Research Council (Advanced Grant 694955-INSEETO). Y. Tokunaga was 
supported by JSPS Grant-in-Aids for Young Scientists (A) (No. 25707032). Y. Tokunaga, Y. Taguchi and Y. Tokura were supported by the Japan Society for the Promotion of Science (JSAP) through its "Funding Program for World-Leading Innovative R\&D on Science and Technology” (FIRST Program). M.F. thanks ETH Zurich and CEMS at RIKEN for support of his research sabbatical. A.C. thanks for supporting his research stay at ETH Zurich.

\section{Author contributions}

All authors contributed to the discussion and interpretation of the experimental results and to the completion of the manuscript. E.H. designed and built the experimental setup and performed the Faraday-rotation microscopy experiments, and proposed the use of magnetic and electric fields to deterministically revive the multiferroic phase inside the domain walls. Y.Z. validated the deconvolution of spatially resolved optical measurements and performed complementary optical experiments. L.K. performed the low-temperature MFM experiments. M.C.W. identified the interconversion of domains and domain walls and coordinated most of the optical experiments. A.B. and A.C. developed the theoretical framework of the interconversion. Y. Tokunaga and Y. Taguchi synthesized the $\mathrm{Dy}_{0.7} \mathrm{~Tb}_{0.3} \mathrm{FeO}_{3}$ samples, coordinated by Y. Tokura. M.F. and T.L. supervised the work.

\section{Competing interests}

The authors declare no competing interests.

\section{Additional information}

Supplementary information The online version contains supplementary material available at https://doi.org/10.1038/s41467-021-22808-7.
Correspondence and requests for materials should be addressed to M.C.W.

Peer review information Nature Communications thanks the anonymous reviewer(s) for their contribution to the peer review of this work.

Reprints and permission information is available at http://www.nature.com/reprints

Publisher's note Springer Nature remains neutral with regard to jurisdictional claims in published maps and institutional affiliations.

(c) (i) Open Access This article is licensed under a Creative Commons Attribution 4.0 International License, which permits use, sharing, adaptation, distribution and reproduction in any medium or format, as long as you give appropriate credit to the original author(s) and the source, provide a link to the Creative Commons license, and indicate if changes were made. The images or other third party material in this article are included in the article's Creative Commons license, unless indicated otherwise in a credit line to the material. If material is not included in the article's Creative Commons license and your intended use is not permitted by statutory regulation or exceeds the permitted use, you will need to obtain permission directly from the copyright holder. To view a copy of this license, visit http://creativecommons.org/ licenses/by/4.0/.

(C) The Author(s) 2021 